\title{
A few-parameter equation of state of the condensed matter and its application to the impact problems
}

\author{
V. Fomin, E. Kraus ${ }^{\text {a }}$ and I. Shabalin
}

Khristianovich Institute of Theoretical and Applied Mechanics, Russian Academy of Science, 4/1 Institutskaya Str., 630090 Novosibirsk, Russia

\begin{abstract}
A simple caloric model of the equation of state is proposed to describe thermodynamic properties of solid materials with the phase transitions being ignored and with the minimum possible number of parameters as the initial data. Thermal oscillations of the crystal lattice are described by the Debye approximation. The values of the parameters on the zero isotherm are calculated analytically from the generalized form of the Gruneisen function. Thermodynamic characteristics are calculated in wide ranges of densities and pressures. Extensive comparisons of theoretical results with experimental data available for high energy densities are performed for the materials considered. Two-dimensional problems of a high-velocity impact of a reactor of a nuclear powerplant with the Earth's surface propulsion system are solved on the basis of Willkins' method with allowance for the equation of state being derived.
\end{abstract}

\section{Equation of state}

We consider a three-term equation of state with the solid-phase free energy being determined as

$$
F(V, T)=E_{x}(V)+c_{v, l} T \ln \left(\frac{\theta(V)}{T}\right)-\frac{1}{2} c_{v, e 0} T^{2}\left(\frac{V}{V_{0}}\right)^{2 / 3}
$$

where $V$ is the specific volume, $E_{x}(V)$ is the "cold" energy, $T$ is the temperature, $c_{v, l}=3 R / A$ is the specific heat of the lattice at constant volume, $A$ is the mean atomic weight, $R$ is the gas constant, $\theta(V)$ is the Debye temperature, and $c_{v, e 0}$ is the experimental value of the electron heat capacity under standard conditions.

The elastic (cold) component of energy $E_{x}(V)$ is related exclusively to interaction forces between the body atoms and is equal (including the energy of zero vibrations) to the specific internal energy at the absolute zero temperature.

The thermodynamic model of a few-parameter equation of state is based on the dependence of the Gruneisen coefficient $\gamma$ on the volume [1]

$$
\gamma(V)=\frac{2}{3}-\frac{2}{\left(1-a V_{0} / V\right)} a=1+\frac{2}{\left(\gamma_{s}-2 / 3\right)}+\frac{2 P_{t, 0}}{K_{s}}
$$

where $\gamma_{s}=\beta K_{s} V_{0} / c_{v}, K_{s}$ is the adiabatic modulus of volume compression, $c_{v}$ is the specific heat at constant volume, and $P_{t, 0}$ is the thermal pressure in the initial state.

The general expression for the volume dependence of the Gruneisen coefficient has the form

$$
\gamma(V)=-\left(\frac{2-t}{3}\right)-\frac{V}{2}\left[\frac{d^{2}}{d V^{2}}\left(P_{x} V^{\frac{2 t}{3}}\right) / \frac{d}{d V}\left(P_{x} V^{\frac{2 t}{3}}\right)\right]
$$

a e-mail: kraus@itam.nsc.ru

This is an Open Access article distributed under the terms of the Creative Commons Attribution-Noncommercial License 3.0, which permits unrestricted use, distribution, and reproduction in any noncommercial medium, provided the original work is properly cited. 


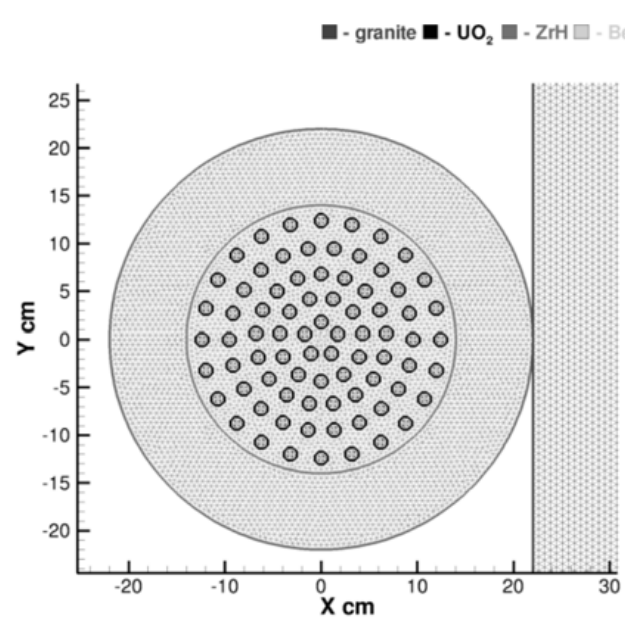

Fig. 1. Reactor with plane geometry.

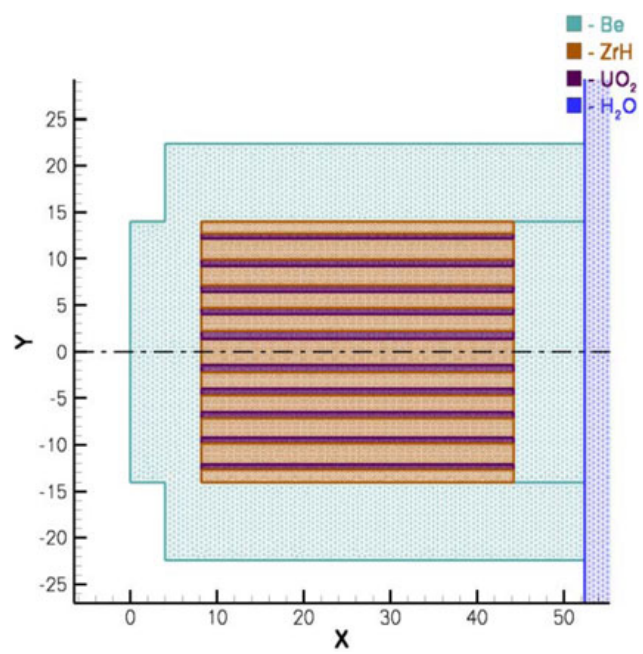

Fig. 2. Reactor with axial geometry.

In Eq. (3), the situation value corresponds to the Landau-Slater theory $[2,3]$ at $t=0$, to the Dugdale-McDonald theory [4] at $t=1$, and to the free-volume theory [5] at $t=2$.

To determine the zero isotherm, we equated the expression for the Gruneisen coefficient (2) at the zero temperature $(T=0 \mathrm{~K})$ to the expression for the generalized Gruneisen coefficient (3):

$$
\frac{2}{3}-\frac{2}{1-a_{x} V_{0} / V}=-\left(\frac{2-t}{3}\right)-\frac{V}{2}\left[\frac{d^{2}}{d V^{2}}\left(P_{x} V^{\frac{2 t}{3}}\right) / \frac{d}{d V}\left(P_{x} V^{\frac{2 t}{3}}\right)\right]
$$

Here, $a_{x}$ is the value of the parameter $\left.a\right|_{T=0}$ at the zero temperature in Eq. (2), which can be taken as $a_{x}=a(0)=1+2 /\left(\gamma_{s}-2 / 3\right)$ as the first approximation.

The differential equation (4) has an analytical solution for "cold" pressure and energy:

$$
P_{x}(V)=C_{1} V^{-2 t / 3}+C_{2} H_{2}(V), \quad E_{x}(V)=-\left(C_{1} V^{1-2 t / 3} /(1-2 t / 3)+C_{2} H_{1}(V)\right)+C_{3}
$$

Using the definition of the Gruneisen coefficient in the Debye approximation $\gamma=-\left.(d \ln \theta / d \ln V)\right|_{T}$ and Eq. (2), we obtain the characteristic Debye temperature on the volume:

$$
\theta(V)=\theta_{0}\left[\frac{\left(a-V / V_{0}\right)}{(a-1)}\right]^{2}\left(\frac{V_{0}}{V}\right)^{\frac{2}{3}}
$$

where $\theta_{0}=\theta\left(V_{0}\right)$ is the Debye temperature at the initial conditions.

The constants for Eq. (5) were determined and calculated in [6]. It was also demonstrated there that the set of semi-empirical relations (1)-(5) describes the behavior of thermodynamic properties of solids within $5-10 \%$ in a wide range of pressures and temperatures. For the equation of state to be applied, it is sufficient to know only six constants $V_{0}, \beta, K_{t}, c_{v}, \Theta_{0}$, and $c_{v, e 0}$ corresponding to the values of these quantities under standard conditions, which can be found in reference books on physical and mechanical properties of substances.

\section{Application of the few-parameter equation of state}

As an example, let us consider a two-dimensional problem of the impact of a nuclear powerplant reactor on the Earth's surface.

In emergency situations, space vehicles "shoot off" the nuclear powerplants. There is a certain probability that the nuclear powerplant reaches the Earth's surface despite considerable thermal and 

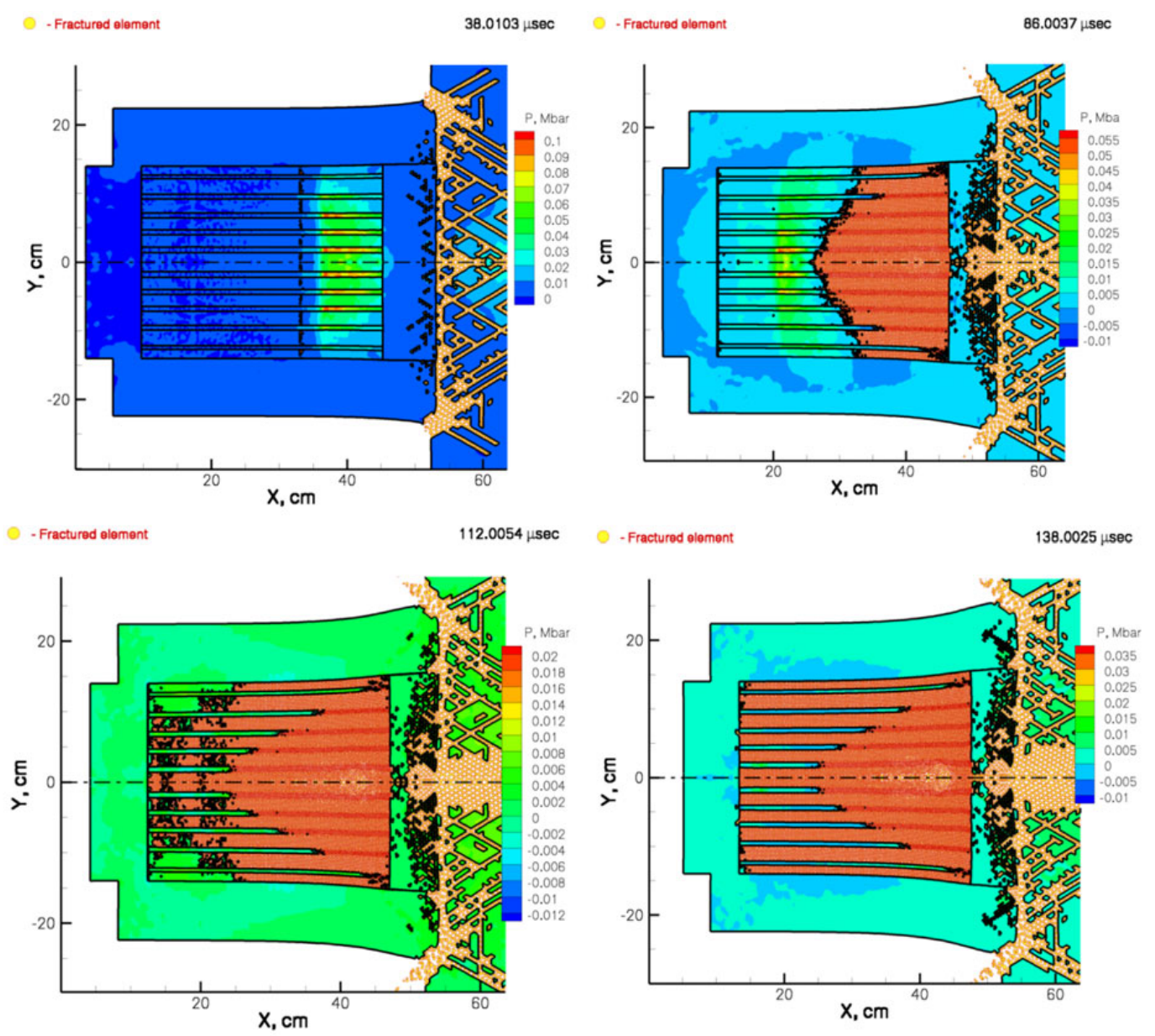

Fig. 3. Impact of a reactor model with a sandstone plate surface.

mechanical loads in dense atmospheric layers. The velocity of the impact of the remaining part of the reactor system can reach $400 \mathrm{~m} / \mathrm{s}$. As the Earth's surface is fairly versatile, the reactor can hit a water surface, rocks, or soft soil.

It is next to impossible to solve impact problems of real engineering objects though the computational engineering development level is rather high and fairly realistic mathematical models of material behavior are available. The reasons are the complicated spatial locations of the reactor fragments and the multiscale character of the problem. In such situations, the object considered is simplified, which makes it possible to construct a number of models aimed at studying the influence of the impact parameters on particular basic fragment of the object.

The problem was solved in a simplified formulation. The simplification used implied that the materials of small-scale parts were averaged in terms of the additive approximation inside the reaction zone (the materials were $\mathrm{Be}, \mathrm{UO}_{2}$, and $\mathrm{ZrH}, 95-97$ wt.\%). It was further assumed that the external elements of the structure burn down when the reactor enters the dense atmospheric layers, and the reactor remainder is an object with a complicated internal structure illustrated in Figs. 1 and 2. The reactor consists of a beryllium shell, uranium dioxide fuel cells, and zirconium hydride fillers. The end-face (longitudinal) and side impacts were considered. In the first case, we have a problem of an impact of the cylinder side surface onto a deformed target (granite and sandstone). A specific feature of this formulation of the problem is a multiply connected computational domain with a large number of contact surfaces. In the second case, the reactor model is formed as a ring-shaped structure, while the computational domain is again multiply connected and numerous contact surfaces are formed. 

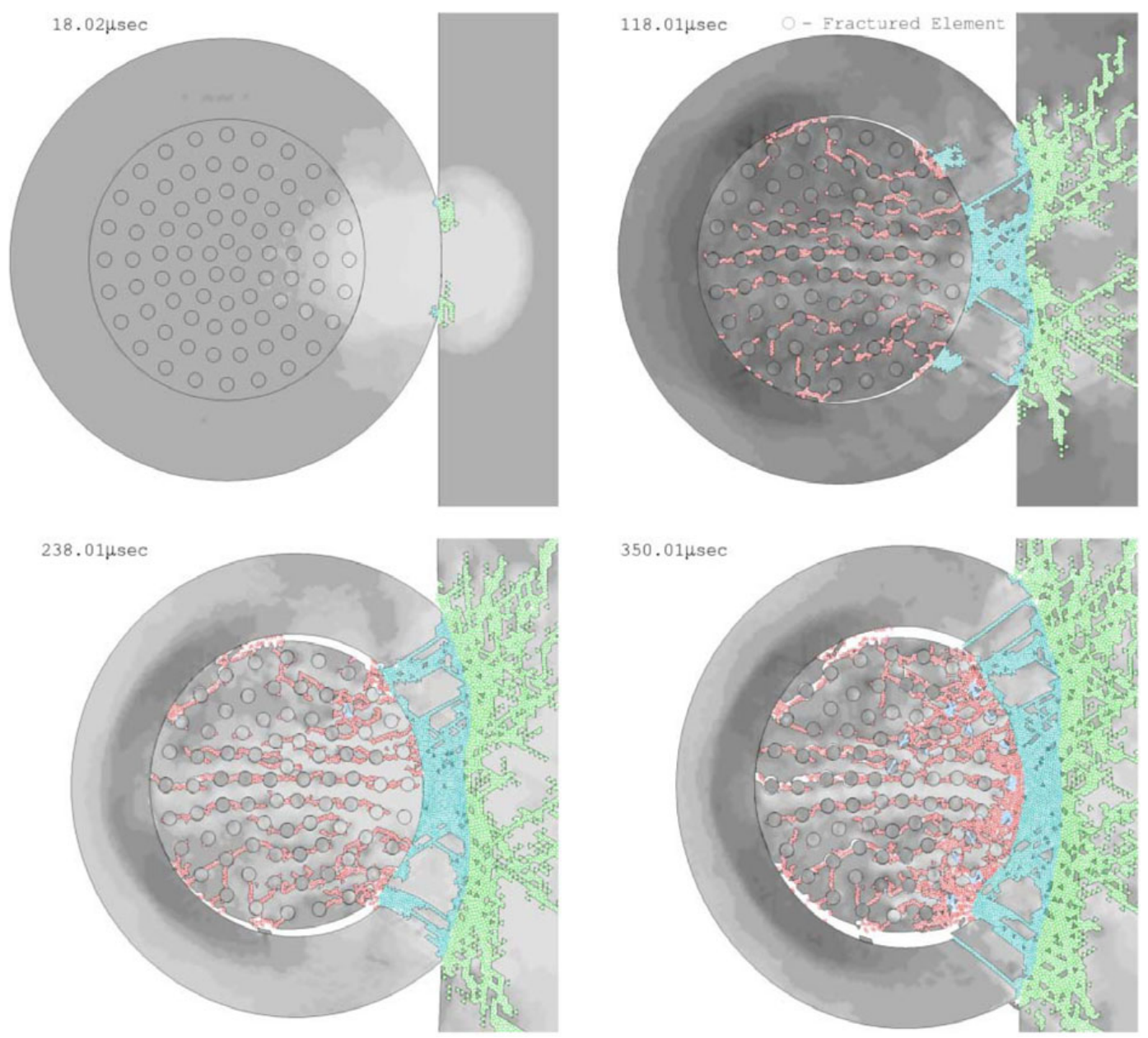

Fig. 4. Frames of the calculated side impact of the reactor model onto the granite plate surface with an impact velocity of $400 \mathrm{~m} / \mathrm{s}$.

Thus, the objective of the work was to study the processes of deformation and fracture of a rather complicated geometric object. The problem was solved in a formulation proposed by Willkins [7] with the use of a symmetric algorithm of contact boundary calculation [8]. Previously, we proposed the process of automatic construction of a triangular grid for an arbitrary multiply connected domain [9].

As the first example, we consider an impact of the reactor on sandstone with the initial velocity of $400 \mathrm{~m} / \mathrm{s}$ in the axial formulation. In the case of the reactor impact onto sandstone, the wave pattern is complicated by the fact that sandstone is destroyed by compressive stresses. This specific feature is induced by the inner structure of sandstone where strong crystals of sand are bound with brittle cement mass. As sand and cement have different compressibilities, the shock wave forms shear stresses on interfaces between the media, which destroy the connections on the boundary, i.e., the resultant product is sand with a fine fraction of cement. Free sand exerts practically no resistance to shear strains.

Thus, the impact compression forms a domain of a fractured material near the reactor-sandstone contact surface and, as a consequence, an unloading wave. The interaction of the side unloading waves and the unloading wave from the fracture zone leads to formation of a zone of tensile stresses with a higher amplitude, leading to fracture of the reactor materials (zirconium hydride filler and fuel cells). Beryllium shell fracture follows the mechanism of shear-induced quasi-static fracture. Figure 3 shows the frames of the impact of the reactor model onto sandstone. A more detailed description of the computations can be found in [10]. 
Let us further consider the computation of the side impact of the nuclear powerplant. Figure 4 shows the frames of the computed side impact of the reactor on a granite plate with an impact velocity of $400 \mathrm{~m} / \mathrm{s}$. This computation shows that the side impact of the reactor is more critical in terms of the impact velocity than the end-face impact because of higher strains in the contact area. While moving, the reactor filler (which is heavier) weighs on the beryllium shell, thus, inducing shear fracture in the contact area with the granite plate and inner fracture of the casing material, which violates the reactor leak-proofness. Numerous cracks appear in the $\mathrm{ZrH}$ filler, which results in disintegration of fuel cells and, as a consequence, possible radioactive contamination of the place of incidence of the reactor. The deformation and the impact are described in more detail in [8].

The work has been financially supported in the frame of the Federal Target Program "Scientific and educational personnel of innovative Russia", state contract P356 from 30.07.2009.

\section{References}

1. A.M. Molodets, Zh. Eksp. Tekh. Fiz. 107, 824 (1995) (in Russian)

2. L.D. Landau, K.P. Stanyukovich, Dokl. Akad. Nauk SSSR 46, 399 (1945) (in Russian)

3. I.C. Slater, Introduction in the Chemical Physics (McGraw Book company, 1935)

4. J.S. Dugdale, D. McDonald, Phys. Rev. 89, 832 (1953)

5. V.N. Zubarev, V.Ya. Vashchenko, Fiz. Tv. Tela 5, 886 (1963) (in Russian)

6. E.I. Kraus, Vestnik NGU. 2, 65 (2007) (in Russian)

7. M. L. Wilkins, Computer Simulation of Dynamic Phenomena (Springer, 1999)

8. V. M. Fomin, A.I. Gulidov, G.A. Sapozhnikov, et al., High-Velocity Interaction of Solids (Publ. SB RAS, 1999) (in Russian)

9. E.I. Kraus, V.M. Fomin, I.I. Shabalin, Vych. Tekhnol. 14, 40 (2009) (in Russian)

10. E.I. Kraus, V.M. Fomin, I.I. Shabalin, Vych. Tekhnol. 11, 104 (2006) (in Russian) 